\title{
A Checkout of the Beam-lines' Beam Position Measurement Systems
}

\author{
Keith Gollwitzer \\ March 15, 2001
}

This pbar note is to describe a recent checkout of the AP3, AP2, AP1 and P2 (F11 to F17) Beam Position Measurement (BPM) systems. The integrity of all BPMs (plates, cables, connectors and RF module) were performed. For each beam-line, the BPM positions polarity were checked: all of the horizontal (vertical) BPMs of a beam-line are to be consistent in defining left/right (up/down) by the sign of the position. The plates' impedances have been measured. The position offsets at the output of the RF modules due to cable and connector differences were determined

Anytime an Energy Doubler BPM RF module (EE-107660) was found not working, a working spare was inserted; three AP2 RF modules were replaced. One connector for one of the cables of BPM728 was repaired. Two new cables were pulled and terminated for BPM716 since there was $>8 \mathrm{~dB}$ difference between the cables. In addition, three digitizer boards were replaced.

\section{Setup and Method}

To test the cable runs and RF modules, a Signal Generator (HP8657A) provided a 53MHz sinusoidal wave. The signal was equally split into matching $1 \mathrm{~dB}$ attenuators. Then each attenuator was connected to a separate cable run, sending identical signals towards the RF module. The electrical amplitudes for the intensity and position provided by the RF module front panel ports were recorded. Then an $1 \mathrm{~dB}$ attenuator was replaced by an $3 \mathrm{~dB}$ attenuator and the intensity and position were recorded. The RF module's outputs were recorded again after the cable runs inputs were switched. The $2 \mathrm{~dB}$ differences should give position results $\pm 0.114 R$, where $R$ is the effective radius of the BPM plates. The observed position amplitude is in $\mathrm{mV}$ and hence a scale factor can be determined for each BPM. To arrive at an electrical offset, the scale factor is then applied to the observed position amplitude when zero is expected.

A BPM's polarity was determined by connecting a signal to only one cable run. The RF module position would be off scale either negatively or positively depending upon which cable run was connected. Along with the RF module's response, the connected cable run and the orientation of the BPM were recorded. There are several possible orientations for a BPM. The connection ports could be located upstream or down-stream of the plates; this was usually determined by which end of a quadrupole the BPM is located. In addition, the ports could be located right/left (above/below) of the beam. Assignments of \pm 1 are made to the BPM's streamness, the ports' orientation with respect to the beam, the connector port to which the used cable is connected, and the position sign observed out of the RF module. It is expected that all BPMs of a plane will have the same overall product: (streamness) (ports' orientation) (cable run used) (position sign). This assumes that the connections of the ports to the plates are uniform.

Each plate's impedance was measured using a Vector Impedance Meter (HP4193A) for a signal at $53 \mathrm{MHz}$. Due to some of the awkward locations of the BPM connection ports for most of the APx beamlines and the length of the meter's probe, an 8ns RG58 cable was used to connect the probe to the ports. For the P2 BPMs only a bnc-tee was used. For a single BPM, the plate impedance measurements were done with and without the $8 \mathrm{~ns}$ cables to determine/check the effect of using the cable. From the results, the 8ns effect was removed and the impedance due to a plate and its inner connections were determined. The impedance difference between the plates was then converted to a position offset. The P2 BPMs are of different varieties and the impedances varied based upon type: unknown amount of inner cabling. The impedance measurements for the pair of plates for each P2 BPM were consistent (same variation as the other beam-line BPMs). The tunnel work was performed J. Budlong and A. Sondgeroth.

\section{Results}

The results are from measurements performed over a two-week period of February 2001. Due to different setups and procedural changes, not all of the quantities are presented. The data collected were entered into Excel spreadsheets. From bpmuti.c, the radius $R=50 \mathrm{~mm}$ has been used to calculate offsets; for 
P1 beam-line BPMs there are some more factors that have been used as in bpmuti.c. See Pbar note 659 for further comments about the scaling used for the APx beam-line BPMs.

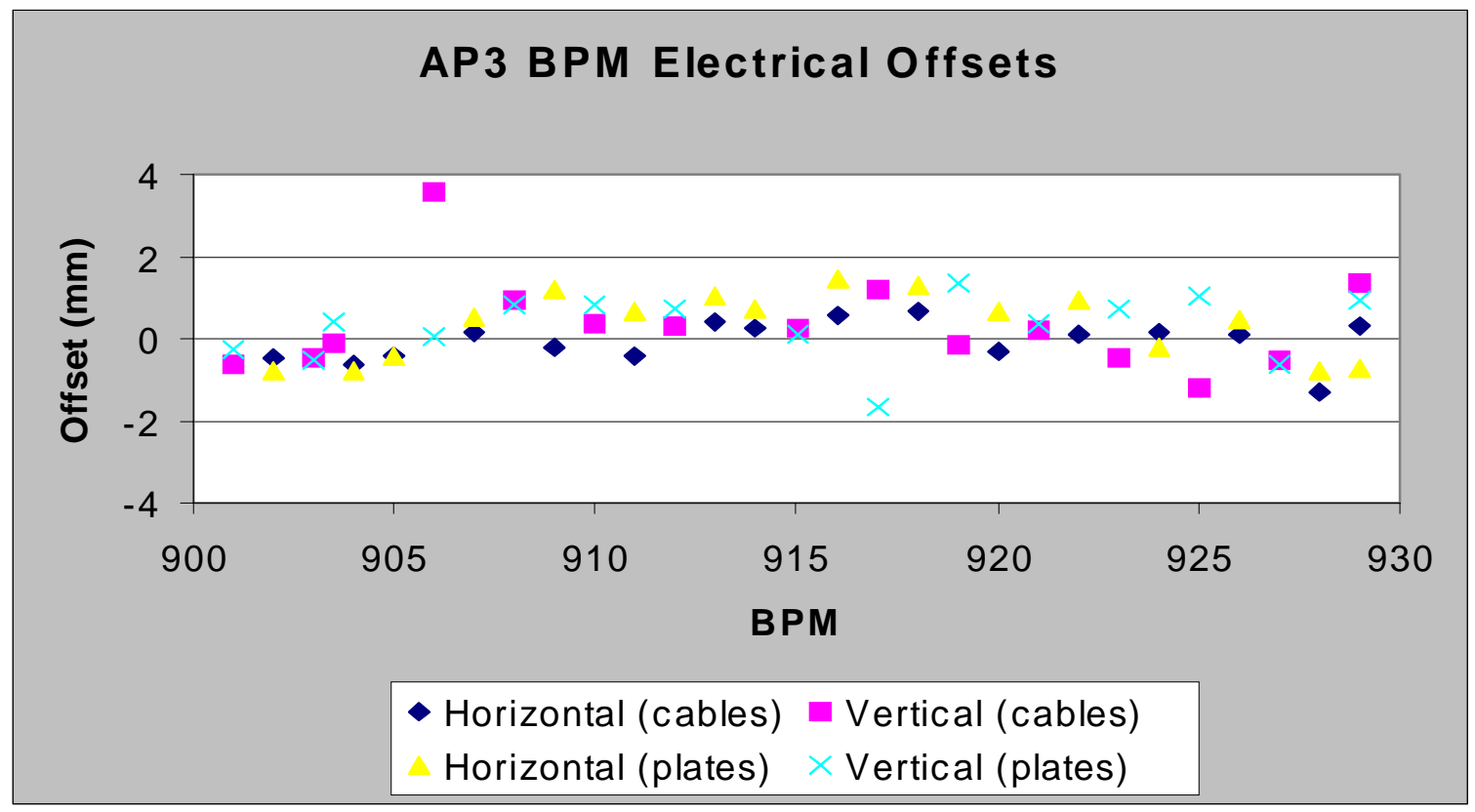

Figure 1: AP3 Electrical Offsets due to the cable runs and pick-up plate differences.

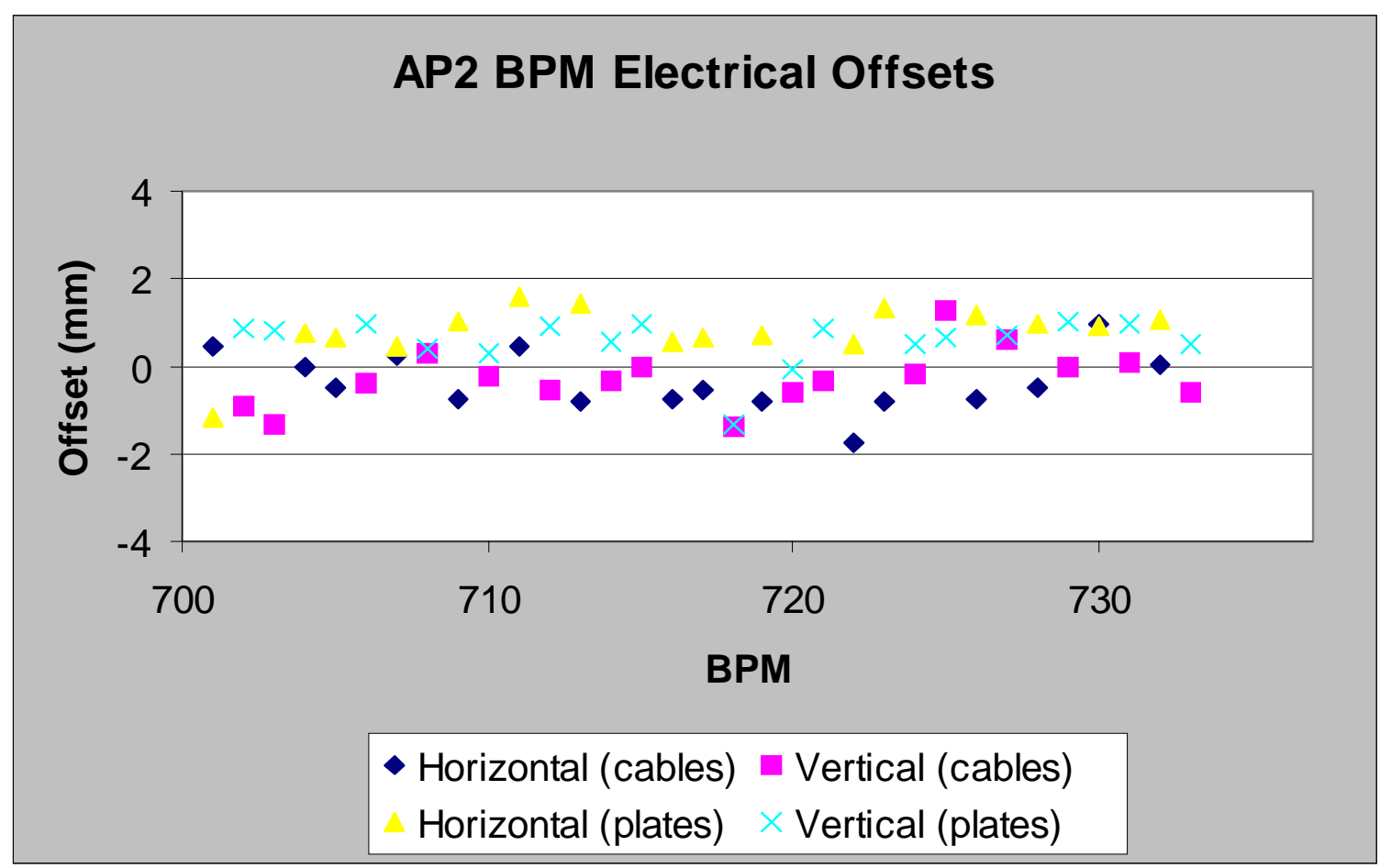

\section{Offsets}

Figure 2: AP2 Electrical Offsets due to the cable runs and pick-up plate differences.

The position offsets, electrical offset and plate impedance differences, are shown in figures 1-4 for the AP3, AP2, AP1 and P2 beam-lines; due to the different BPM types in P2 and the variety of impedance values, no P2 plate impedance results are shown. The offsets determined by plate impedance differences are comparable to the expected millimeter resolution. Most of the cable runs' electrical offsets are also 
comparable to the expected resolution. There are a few BPMs where the electrical offsets are greater than a few millimeters due to differences of the cable runs attenuation (a few $\mathrm{dB}$ ).

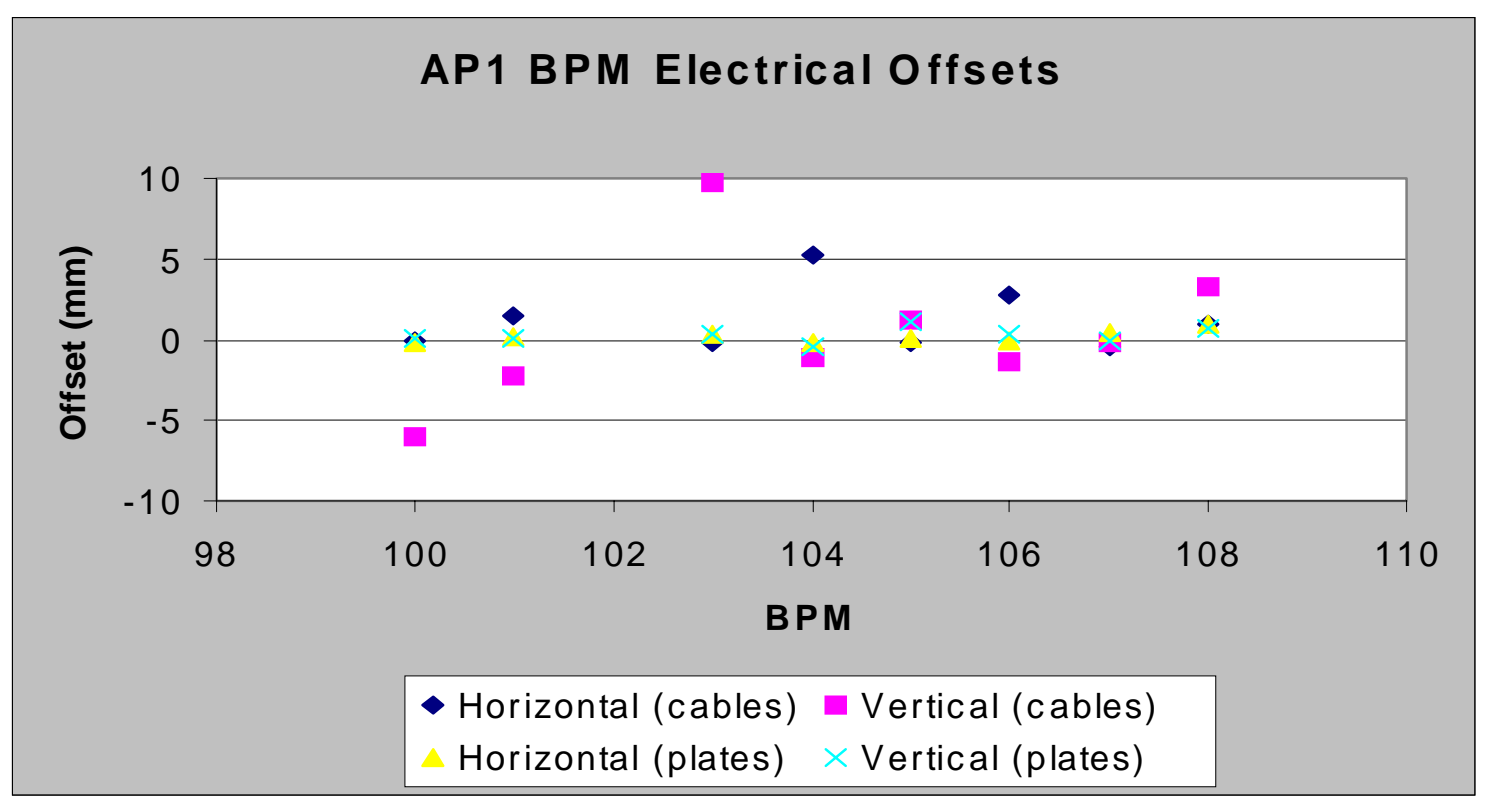

Figure 3: AP1 Electrical Offsets due to the cable runs and pick-up plate differences.

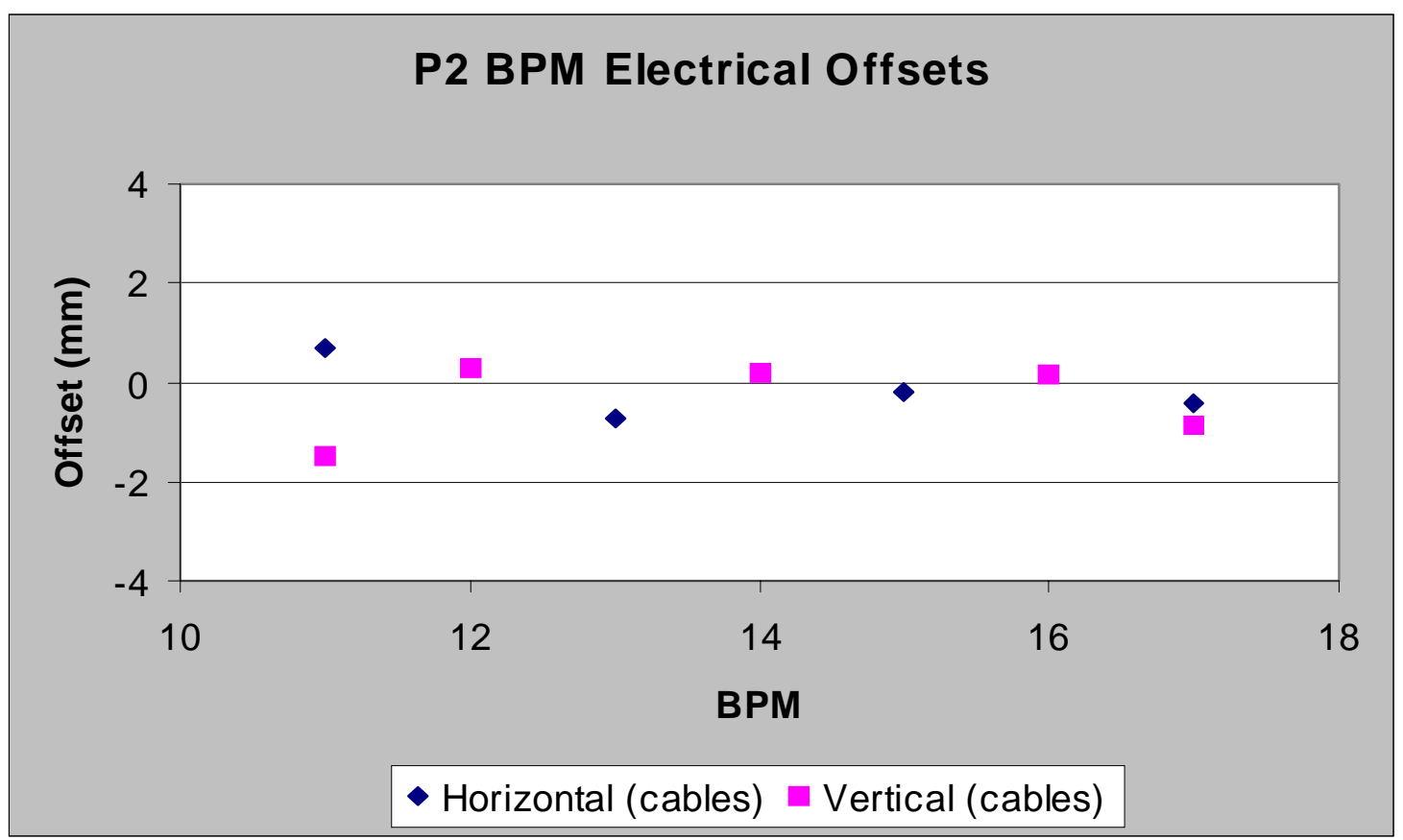

Figure 4: P2 Electrical Offsets due to the cable runs.

\section{Intensities}

As a benefit of checking each set of cable runs with a consistent signal, the intensity readings can be relatively calibrated. The differences between BPMs' intensities are due to the different length cable runs and the quality of cable used. Tables 1 and 2 provide the ratios of each BPM intensity signal to the 
largest intensity recorded for the AP2 and AP3 beam-line, respectively. The other beam-lines were not done using a consistent signal and/or measurement technique.

Table 1: Relative AP2 BPM Intensities.

\begin{tabular}{|r|l|l|l|l|l|l|l|}
\hline H701 & 0.39 & V710 & 0.79 & H719 & 0.26 & V727 & 0.57 \\
\hline V702 & 0.38 & H711 & 0.68 & V720 & 0.29 & H728 & 0.66 \\
\hline V703 & 0.42 & V712 & 0.56 & V721 & 0.30 & V729 & 0.81 \\
\hline H705 & 0.49 & H713 & 0.49 & H722 & 0.33 & H730 & 0.80 \\
\hline V706 & 0.51 & V714 & 0.44 & H723 & 0.33 & V731 & 0.93 \\
\hline H707 & 0.59 & H716 & 0.52 & V724 & 0.38 & H732 & 1.00 \\
\hline V708 & 0.62 & H717 & 0.23 & V725 & 0.42 & V733 & 0.93 \\
\hline H709 & 0.73 & V718 & 0.22 & H726 & 0.50 & & \\
\hline
\end{tabular}

Table 2: Relative AP3 BPM Intensities.

\begin{tabular}{|r|l|l|l|r|l|r|l|}
\hline V901 & 0.93 & V908 & 0.98 & H916 & 0.59 & H924 & 0.47 \\
\hline H202 & 0.97 & H909 & 0.69 & V917 & 0.52 & V925 & 0.50 \\
\hline V703a & 0.94 & V910 & 0.89 & H918 & 0.51 & H926 & 0.23 \\
\hline V703b & 1.00 & H911 & 0.80 & V919 & 0.40 & V927 & 0.26 \\
\hline H904 & 0.96 & V912 & 0.75 & H920 & 0.48 & H928 & 0.23 \\
\hline H905 & 0.85 & H913 & 0.70 & V921 & 0.67 & & \\
\hline V906 & 0.94 & H914 & 0.66 & H922 & 0.36 & & \\
\hline H907 & 0.91 & V915 & 0.60 & V923 & 0.56 & & \\
\hline
\end{tabular}

\section{Polarity}

The AP2 BPMs' polarities are found to be consistent. Beam studies confirmed that all AP1 BPM's polarities are consistent. It appears that all of the P2 BPM polarities are consistent.

The AP3 vertical BPMs are consistent. The polarities of AP3 BPMs 902H, 904H, 926H, 928H and $929 \mathrm{H}$ were different than the rest of the AP3 beam-line. The first two are located above the accumulator and the latter three are located in the pre-target enclosure. One could believe that the cable run connections for these BPMs were done at a different time than the rest of the beam-line that definition of left/right for these BPMs could be different: accumulator or AP1 like. In fact, the cable run labels support that this. The connection of the cable runs to the RF modules have been switched and documented. Now all of the horizontal AP3 BPMs have the same polarity.

\section{Conclusions}

The BPM systems of the beam-lines have been tested and repaired as necessary. It is believed that all BPMs of one plane have the same polarity. For each BPM, the plates show consistent impedances. The electrical offsets have been determined assuming that the current software sets of effective plate radii are correct in bpmuti.c. Hence, the electrical offsets presented here are consistent with the present measurement system. 